\title{
PERFIL CLÍNICO-EPIDEMIOLÓGICO DE CÂNCER DE BOCA EM IDOSOS
}

\section{CLINICAL AND EPIDEMIOLOGICAL PROFILE MOUTH CANCER IN THE ELDERLY}

\section{PERFIL CLÍNICO Y EPIDEMIOLÓGICO CÁNCER DE BOCA EN PERSONAS MAYORES}

\author{
Rafael de Medeiros Vasconcelos ${ }^{1}$ \\ Jaleska Santos Olinto Trindade ${ }^{2}$ \\ Izabel Cristina Pinheiro de Almeida ${ }^{3}$ \\ Ricardo José Curioso da Silva ${ }^{4}$ \\ Maria de Lourdes Silva de Arruda Morais ${ }^{5}$
}

\section{RESUMO}

O Câncer de boca é o oitavo tipo de câncer mais comum no mundo. Nosso objetivo foi avaliar a mortalidade por câncer de boca em idosos do estado do Rio Grande do Norte-RN e traçar o perfil destes pacientes. Realizou-se nos arquivos da LNRCC um levantamento epidemiológico dos casos de câncer de boca em pacientes maiores de 60 anos, no período de janeiro/05 a dezembro/09, um estudo observacional do tipo descritivo e retrospectivo. Foram selecionados 325 pacientes, onde 235 encontravam-se vivos com idade média de 74 anos. Destes, 181 casos eram do sexo masculino. A História familiar de câncer ocorreu em 14\% dos pacientes. Tabagismo esteve presente em $72 \%$ dos casos e o tempo médio de exposição direta foi de 49,6 anos. Etilismo ocorreu em $36 \%$ dos casos, com tempo médio de exposição de 41,9 anos. A maioria dos pacientes apresentava carcinoma espinocelular medianamente diferenciado. Os sítios primários de maior prevalência foram lábio e língua. 32\% dos pacientes realizaram apenas cirurgia e $24 \%$ dos pacientes realizaram cirurgia, radioterapia e quimioterapia. Quanto a evolução clínica, 26\% dos pacientes foram a óbito, $41 \%$ de cura/seguimento e $33 \%$ sem informação. Nossos achados corroboram com a literatura.

Palavras-chave: Carcinoma Espinocelular, Idosos, Epidemiologia.

\footnotetext{
${ }^{1}$ Acadêmico do curso de medicina da Universidade Federal do Rio Grande do Norte - UFRN

${ }^{2}$ Bióloga e mestranda do curso de Biotecnologia da UNP

3 Médica cirurgiã de cabeça e pescoço da Liga Norte Rio Grandense Contra o Câncer - LNRCC

${ }^{4}$ Médico cirurgião de cabeça e pescoço da Liga Norte Rio Grandense Contra o Câncer - LNRCC

${ }^{5}$ Odontóloga, Doutora pesquisadora da Liga Norte Rio Grandense Contra o Câncer - LNRCC e professora do mestrado em Biotecnologia da UNP
} 


\begin{abstract}
The oral cancer is the eighth most common cancer in the world. Our goal is to evaluate the oral cancer mortality among the elderly in the state of Rio Grande do Norte, RN, and trace the profile of these patients. This was in the archives of HLA-LNRCC an epidemiological survey of cases of mouth cancer in patients over 60 years, from the January/05 December/09, a descriptive retrospective observational study.: We selected 325 patients, 235 live and 90 deaths. The average age of patients was 74 years. 181 cases were male and 144 female cases. Family history of cancer occurred in 14\% of patients. Smoking was present in $72 \%$ of cases, and mean duration of direct exposure was 49.6 years. Alcoholism occurred in $36 \%$ of cases, with mean exposure of 41.9 years. Most patients had squamous cell carcinoma moderately differentiated. The most prevalent primary sites were the lip, tongue and oropharynx. $32 \%$ of patients underwent only surgery, and $24 \%$ of the patients underwent surgery, radiotherapy and chemotherapy. In clinical outcome was found $26 \%$ of deaths, $41 \%$ cure / follow-up and $33 \%$ dropout / not informed. Our findings corroborate the literature.
\end{abstract}

Keywords: squamous cell carcinoma, elderly, epidemiology

\title{
RESUMEN
}

El cáncer de boca es el octavo cáncer más común en el mundo. Nuestro objetivo fue evaluar la mortalidad por cáncer bucal entre los adultos mayores en el estado de Rio Grande do Norte, RN y el perfil de estos pacientes. Conservados en los archivos de LNRCC datos epidemiológicos sobre casos de cáncer de boca en pacientes mayores de 60 años, a partir de la enero/05 diciembre/09, observacional con una extensión. Descriptivo y retrospectivo Se seleccionaron 325 pacientes, en 235 estaban vivos con una edad media de 74 años. De ellos, 181 casos fueron varones. Una historia familiar de cáncer ocurrió en el 14\% de los pacientes. Fumar estuvo presente en el 72\% de los casos y el tiempo promedio de exposición directa fue de 49,6 años. El alcoholismo se produjo en el 36\% de los casos, con una exposición media de 41,9 años. La mayoría de los pacientes habían diferenciado moderadamente carcinoma de células escamosas. Los sitios primarios fueron el labio y la lengua predominante. 32\% de los pacientes fueron sometidos a cirugía y sólo el $24 \%$ de los pacientes fueron sometidos a cirugía, la 
radioterapia y la quimioterapia. En cuanto a la evolución clínica, el 26\% de los pacientes falleció, el $41 \%$ de curación / seguimiento de la información y el 33\% fuera. Nuestros resultados corroboran la literatura.

Palabras clave: Carcinoma de células escamosas, Personas Mayores, Epidemiologia.

\section{INTRODUÇÃO}

O câncer oral responde por quase 3\% dos casos de câncer no mundo, representado em 90 a $95 \%$ dos casos pelo carcinoma epidermoide(1). No Brasil são estimados, para o ano de 2012, 10 novos casos a cada 100 mil homens e 4 novos casos a cada 100 mil mulheres, sendo o quinto tipo tumoral mais comum em homens. No Rio Grande do Norte foram estimados para o ano de 2012, 190 novos casos(2).

Predominantemente o câncer de boca ocorre em idosos do sexo masculino, da zona rural, com baixo nível socioeconômico(3), pelo fato destas características conferirem maior exposição aos agentes carcinógenos. A etiologia do câncer de boca está relacionada com a interação entre fatores intrínsecos do hospedeiro e exposição ambiental, tais como fumo(4), dieta e infecções por HPV.

O carcinoma epidermóide oral exibe elevadas taxas de mortalidade, mesmo com o avanço das modalidades terapêuticas, o que se atribui principalmente à resposta variada ao tratamento e a falha no diagnóstico precoce(5).

Nosso estudo reveste-se de especial importância pelo fato de apresentar o perfil epidemiológico das neoplasias de cavidade oral em um grupo especial de pacientes: os idosos.

Os objetivos do presente estudo constituem em avaliar a mortalidade por câncer de boca no idoso no estado do Rio Grande do Norte-RN, bem como traçar o perfil dos pacientes portadores desta patologia.

\section{MÉTODOS}

Para realização do estudo, foi realizado nos arquivos da Liga Norte Riograndense Contra o Câncer, centro de referência do Estado para tratamento de câncer, um levantamento dos casos de câncer de boca, em pacientes acima de 60 anos de idade, que ocorreram de janeiro/05 a dezembro/09, 
através de estudo observacional do tipo descritivo, retrospectivo, longitudinal e de abordagem qualitativa e quantitativa.

Para tanto foram coletados destes registros dados clínicos e epidemiológicos, incluindo idade, etnia (cor da pele), sexo, profissão, naturalidade, procedência, história familiar de câncer e respectivo(s) grau(s) de parentesco, hábitos, diagnóstico histopatológico, sítio primário do tumor, estadiamento clínico, tratamento(s) realizado(s) e evolução clínica. Os dados coletados foram analisados através de Análise Descritiva.

\section{RESULTADOS}

Foram avaliados 325 pacientes, sendo 235 vivos e 90 óbitos. A idade média dos pacientes foi de $74 \pm$ 9,6 anos, cuja distribuição por sexo foi de 181 casos para o sexo masculino e 144 do sexo feminino.

História familiar de câncer esteve presente em 17\% $(\mathrm{n}=56)$ dos pacientes, ausente em 22\% $(n=70)$ deles, e não informada em 61\% $(n=199)$ dos casos, conforme observado no gráfico 1.

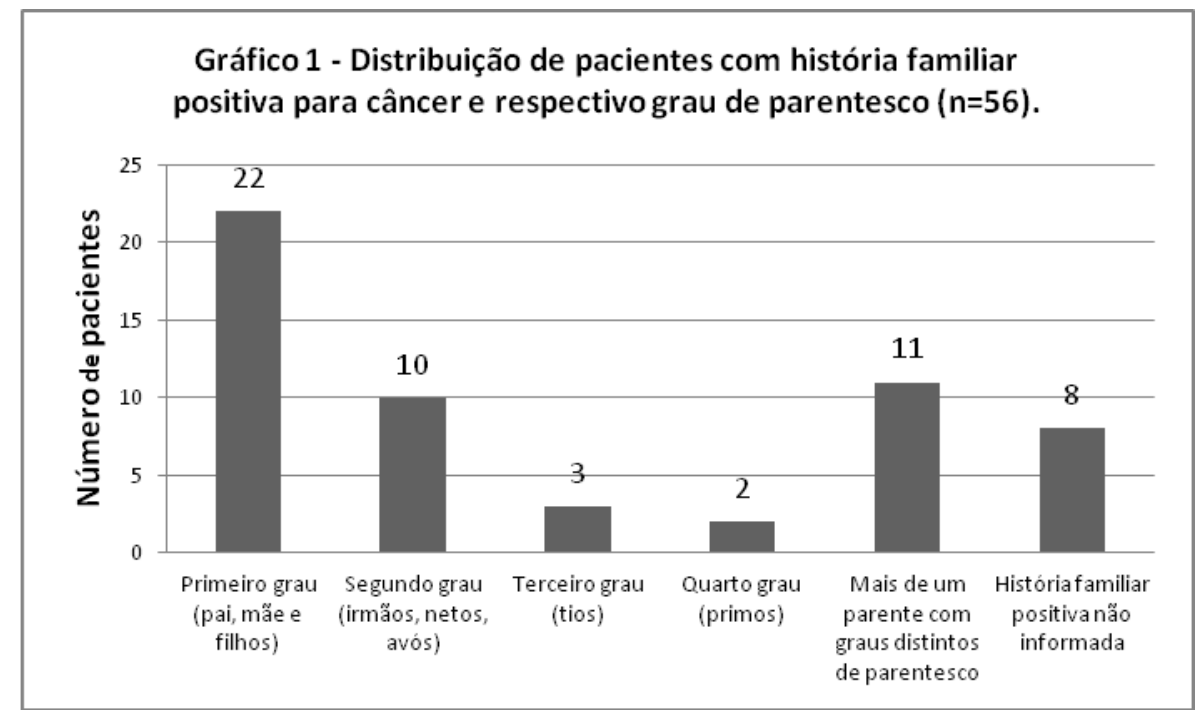


Tabagismo esteve presente em $72 \%(n=235)$ dos casos, ausente em $6 \%(n=19)$ dos casos e não informado em $22 \%(n=71)$ dos casos. Destes, 85 pacientes fumavam apenas cigarro, 67 fumavam apenas cachimbo, 44 fumavam apenas fumo brejeiro/de rolo, 04 fumavam apenas cigarro de palha, 06 fumavam mais de um tipo de tabaco, e nenhum dos pacientes fumava outro tipo de tabaco. O tempo médio de exposição direta ao tabagismo, em anos, foi de 49,6 \pm 16,1 anos. O Etilismo esteve presente em $37 \%(n=120)$ dos casos, ausente em $25 \%(n=83)$ dos casos e não informado em $38 \%(n=122)$ dos casos. O tempo médio de exposição ao etilismo, em anos, foi de 41,9 \pm 14,0 anos.

O Carcinoma espinocelular (CEC) do tipo medianamente diferenciado representou $80 \%$ dos diagnósticos histopatológicos. Quanto ao sítio primário do tumor observou-se amplo perfil de distribuição, sendo os mais prevalentes o lábio e língua, conforme gráfico 2.

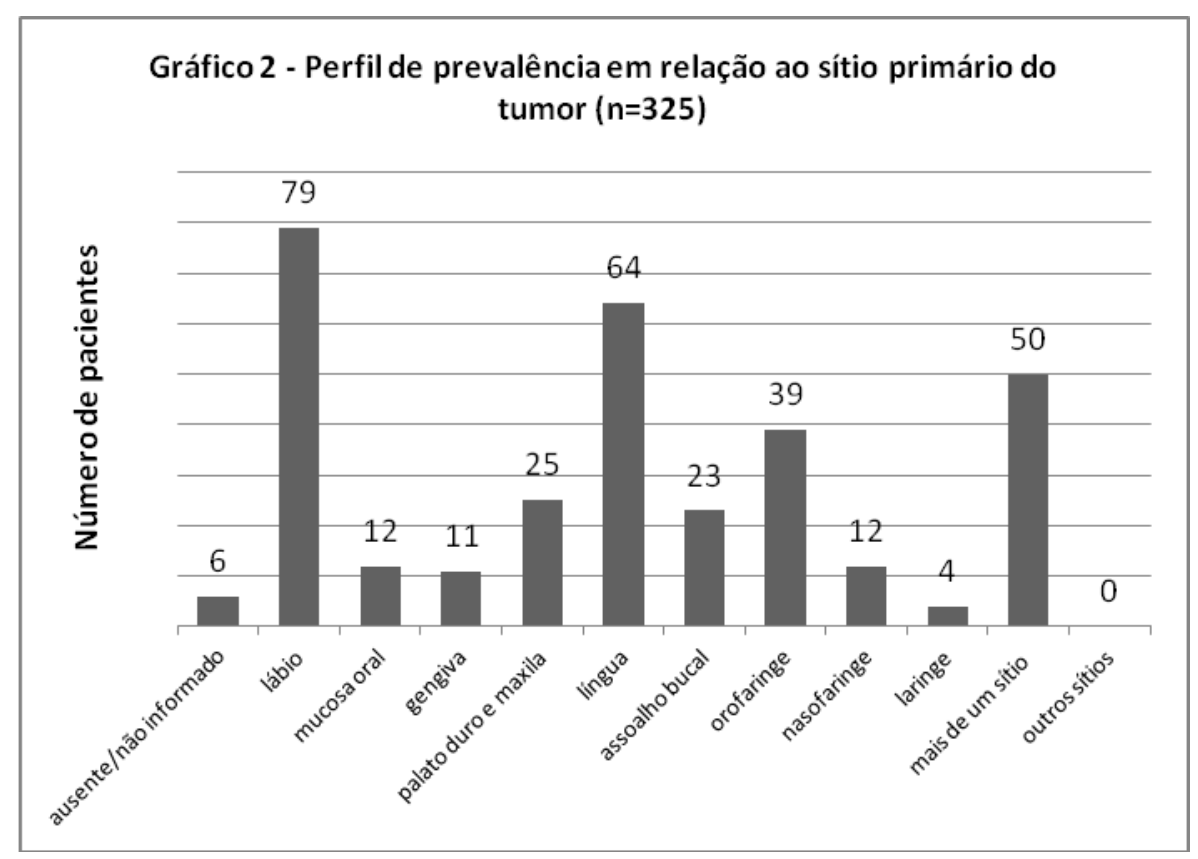

Todas as formas utilizadas como tratamento, isoladas ou em associação, estão descritas no gráfico 3. 


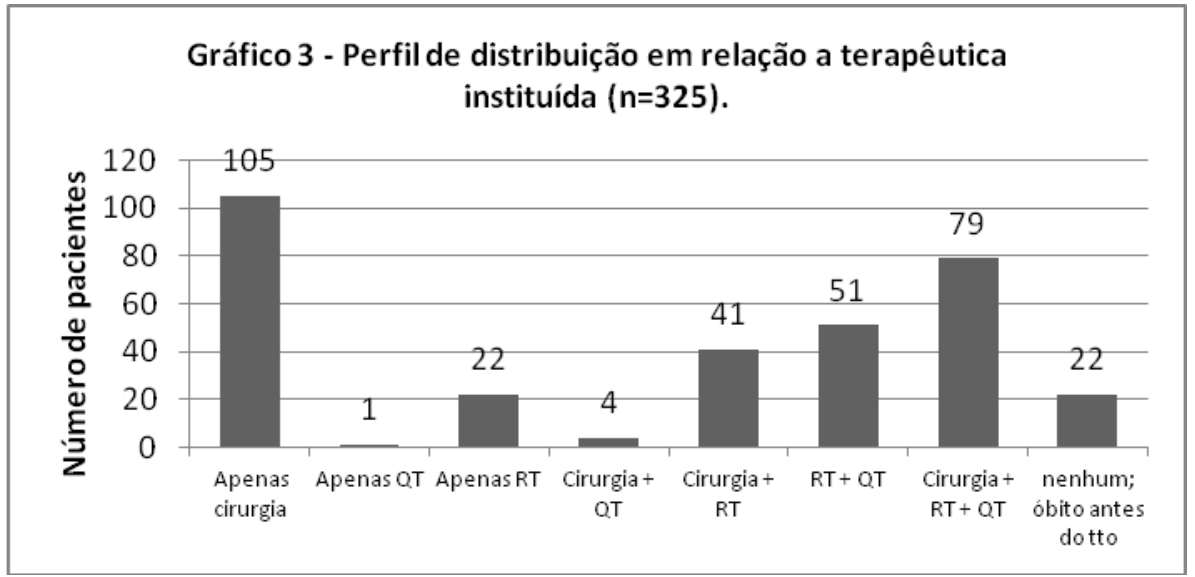

Foram considerados, como critérios de evolução clínica dos pacientes: óbito, cura/seguimento e abandono/não informado. Sendo assim, foi encontrado o seguinte perfil: $26 \%$ de óbitos, $41 \%$ de cura/seguimento e $33 \%$ de abandono/não informado.

\section{DISCUSSÃO}

A faixa etária mais atingida situa-se entre 60 e 80 anos, e embora a maioria dos estudos ressalte uma ampla redução na sobrevida global(6), nossa casuística demonstrou apenas $26 \%$ de óbitos em idosos. Os dados encontrados mostram pouca predileção entre os sexos, diferindo da literatura, que evidencia grande distinção nos perfis de incidência e prevalência entre os sexos, sendo os do sexo masculino os mais acometidos na maior parte dos estudos(7).

A hereditariedade é um fator a ser considerado, pois encontramos $17 \%$ dos pacientes com história familiar de câncer, especialmente em parentes de primeiro grau. Entretanto, há poucos estudos evidenciando tal associação(8). Além disso, não havia informação sobre os tipos tumorais encontrados nos familiares.

O uso crônico de tabaco e de álcool confirmaram-se, em acordo com a literatura(9), como os principais fatores de risco para câncer de boca, e quando usados de forma associada, demonstram efeito sinérgico dose-dependente na carcinogênese, segundo Petti(10). 
É preciso buscar campanhas de educação continuada no intuito de orientar a população quanto aos fatores de risco e sintomatologia precoce associada a doença, bem como de manter uma higiene e saúde bucais adequadas.

\section{CONCLUSÃ O}

Os resultados obtidos mostram o perfil dos idosos acometidos por câncer de boca no Estado, sendo possível concluir que estímulos ambientais são os principais fatores de risco para o surgimento deste grupo de neoplasias, embora outros fatores possam ser levados em consideração, tais como a hereditariedade. Nesse ponto de vista, aspectos clínicos e epidemiológicos são fundamentais para a elaboração de estratégias de prevenção, diagnóstico e abordagens terapêuticas adequadas.

\section{REFERÊNCIAS:}

1. Neville BW; Day TA. Oral cancer and precancerous lesions. CA Cancer J Clin. 2002; 52:195215.

2. Brasil. Ministério da Saúde. Instituto Nacional de Câncer. Estimativa 2012: Incidência de câncer no Brasil/Instituto Nacional de Câncer./ - Rio de Janeiro: INCA, 2011.

3. Borges FT, Garbin CAS, Carvalhosa AA, Castro PHS, Hidalgo LRC. Epidemiologia do câncer de boca em laboratório público do Estado de Mato Grosso, Brasil. Cad. Saúde Pública, Rio de Janeiro, set 2008; 24(9):1977-1982.

4. Proia NK, Paszkiewicz GM, Nasca MAS, Franke GE, Pauly JL. Smoking Smokeless TobaccoAssociated Human Buccal Cell Mutations and Their Association with Oral Cancer - A Review. Cancer Epidemiol Biomarkers Prev, June 2006; 15(6).

5. CHOI, K.K et al. Independent prognostic factors of 861 cases of oral squamous cell carcinoma in Korean adults. Oral Oncol. 2006; 42(2):208-17. 
6. Warnakulasuriya S. Living with oral cancer: Epidemiology with particular reference to prevalence and life-style changes that influence survival. Oral Oncology 2010; 46:407-410

7. C.Scully, J. Bagan. Oral squamous cell carcinoma review. Oral Oncology 2009;45:301-308.

8. Pereira LA, Sobrinho JA, Rapoport A, Dedivitis RA. Epidemiologia do câncer bucal em Barretos, São Paulo. Rev Bras Cir Cab Pesc. Abr/Mai/Jun 2003;31(2): 35-39.

9. P.E. Petersen. Oral cancer prevention and control - The Approach of the World Health Organization. Oral Oncology 2009 Apr-May;45(4-5):454-460.

10. S. Petti. Lifestyle risk factors for oral cancer. Oral Oncology 2009; 45:340-350.

Autor Responsável: Maria de Lourdes Silva de Arruda Morais Endereço: Liga contra o Câncer/DEPECOM Av. Miguel Castro, 1355. Bairro Dix-Sept Rosado. CEP 59062-000. Natal/RN E-Mail: lourd_arruda@ hotmail.com 